\title{
LANCASTER MOOR HOSPITAL IN THE 1930s FROM THE ARCHIVES
}

\author{
At the beginning of the nineteenth century, legislation empowered local authorities to make provision for the detention and \\ treatment of insane persons.
}

Five acres of land on the Moor of Lancaster were purchased, plans prepared and approved, and building commenced. The first sixty patients were admitted in July 1816 with a further sixty-three in the following year. Demand was such that additional accommodation was provided in 1824 and 1855, and yet further extensions over the next twenty years. Finally, land on the other side of Quernmore Road was purchased to house 825 or so more patients. The Ladies' Villa (now Ridge Lea) opened in 1916, when the total estate covered over 200 acres. By 1938 the estate had increased to nearly 400 acres.

The revolutionary reforms in the treatment of the insane introduced by Tuke at York about 1800 were either unknown or ignored at the Lancaster Asylum when it first opened. Patients were permanently incarcerated in cells or fastened to fixed seats in rooms maintained at high temperatures by steam. Sanitary requirements were met by an open gutter running through rooms. Measures of restraint included manacles, straps, muzzles and leather straight jackets.

Despite more wards and extensions in the inter-war years, overcrowding continued and inpatients numbers were always around 3,000 .

\section{EXTRACTS FROM THE REPORT OF THE COMMISSIONERS OF THE BOARD OF CONTROL, MARCH 1938}

\begin{abstract}
"We feel, bearing in mind the many handicaps which occur in overcrowded conditions, that the wards of the Hospital are in general well kept and comfortably furnished. Efforts have been made to brighten many of the wards with pot plants and flowers and steps have been taken to replace backless benches with chairs. A considerable number of day rooms, dormitories and single rooms are in need of decoration and we were pleased to see that where this has been started, bright colours have been employed. The patients' clothes were neat and tidy and progress is being made by introducing modern underclothes for suitable patients. An additional steam press would facilitate the pressing of the male patients' suits.
\end{abstract}

The weekly maintenance charge per head for home patients is $20 \mathrm{~s} 5 \mathrm{~d}$ and that for private patients ranges from $24 \mathrm{~s} 6 \mathrm{~d}$ to $45 \mathrm{~s} 6 \mathrm{~d}$. The average weekly maintenance cost as last ascertained is $19 \mathrm{~s} 7 \mathrm{~d}$. There are 446 Private Patients (162 M, $284 \mathrm{~F}$ ) of whom 66 belong to the service or ex-service class.

There is no doubt that the erection of an admission hospital will promote a rise in the number of voluntary admissions. The present unfavourable environment of the ward for new female admissions is hardly calculated to increase the use of the voluntary principle.

The high standard of medical care of the patients in this Mental Hospital is indicated by the use in so many directions of the advantages of modern and progressive medicine, firstly by the comprehensive lines of investigation of new admissions and secondly in the matter of treatment. Careful physical and psychological examinations are conducted: a very complete blood examination, and, where required, an examination of the cerebral spinal fluid are carried out as well as routine bacteriological examination of excreta. As a result of the blood investigations as many as 16 cases of pernicious anaemia are now under treatment. Each new patient receives a dental overhaul.

In the matter of treatment of both physical and mental states, up-to-date methods are employed. Convulsive shock treatment has been adopted here since October last. Much relief has resulted from the high atropine treatment of the sequelae of Encephalitis Lethargica. The use of sodium amytal has rendered certain inaccessible patients amenable to examinations and treatment. Blood bromism in new admissions has been investigated with profit to certain patients. Malarial inoculation and other modes of treatment for general paralysis have been applied here for many years.

The hospital now possesses apparatus for X-ray examination and treatment, for ultra-violet radiation and other special physical treatments. The public health requirements of this large community receive particular attention, new admissions and staff being protected by T.A.B. injections, which are repeated annually in the cases of the personnel and patients employed on the sanitary duties of the Hospital, the staff and the wards where former typhoid patients still require segregation and, thirdly, patients of faulty habits. All garments used in isolation wards are passed through the autoclave prior to their handling in the laundry. Specially coloured overalls and bed jackets are made in the sewing room for wear in these wards. A distinctive overall and apron for all engaged in the main kitchens and dining halls is also provided.

One valuable method of treatment, which is made use of to a certain extent, is that of occupation by handcrafts and other means but its organisation has so far been iittle advanced and considerable numbers of patients yet remain outside its scope. We realize that Dr Silverston is familiar with the lines of its appropriate development and that he is taking steps to secure personnel and accommodation. A kindred treatment by physical exercises is utilised for women patients but the men 
have not yet had the advantage of this method of treatment or of associated gymnastic exercises.

At the time of our visit $12 \%$ of the patients on each side were under treatment in bed, a considerable proportion being advanced in years. Three men and eleven women are receiving treatment for tuberculosis under conditions calculated to improve their state and to prevent the spread of this infection. In view of the indirect ventilation of many side rooms and other quarters it is a matter of good fortune that so few cases of this disease occur here. It is noted, however, that 22 patients have died of tuberculosis in the last 13 months. The co-operation in the examination of suspected cases by the Tuberculosis Officers of the County Council is much appreciated. second vegetable at dinner and it is considered, we understand, that this has reduced the amount of gastrointestinal trouble. The ordinary dietary is drawn up so as to afford a possible three or four variations for any one day; its use is therefore equivalent to a three to four weekly rota for the dinner menus and there is weekly rotation of breakfasts and teas. A second course is served at each dinner unless the first course be of meat pie. The milk is obtained on contract for the greater part of the Hospital but the Private Ladies Villa is supplied by the Hospital Farm, where the herd is exceptionally well groomed. The means however for cleaning and disinfecting the utensils are only on a domestic scale; though they are treated with hot water twice a day they are only boiled once a week. It is desirable to replace the pail handles by those which fall beyond the rim.

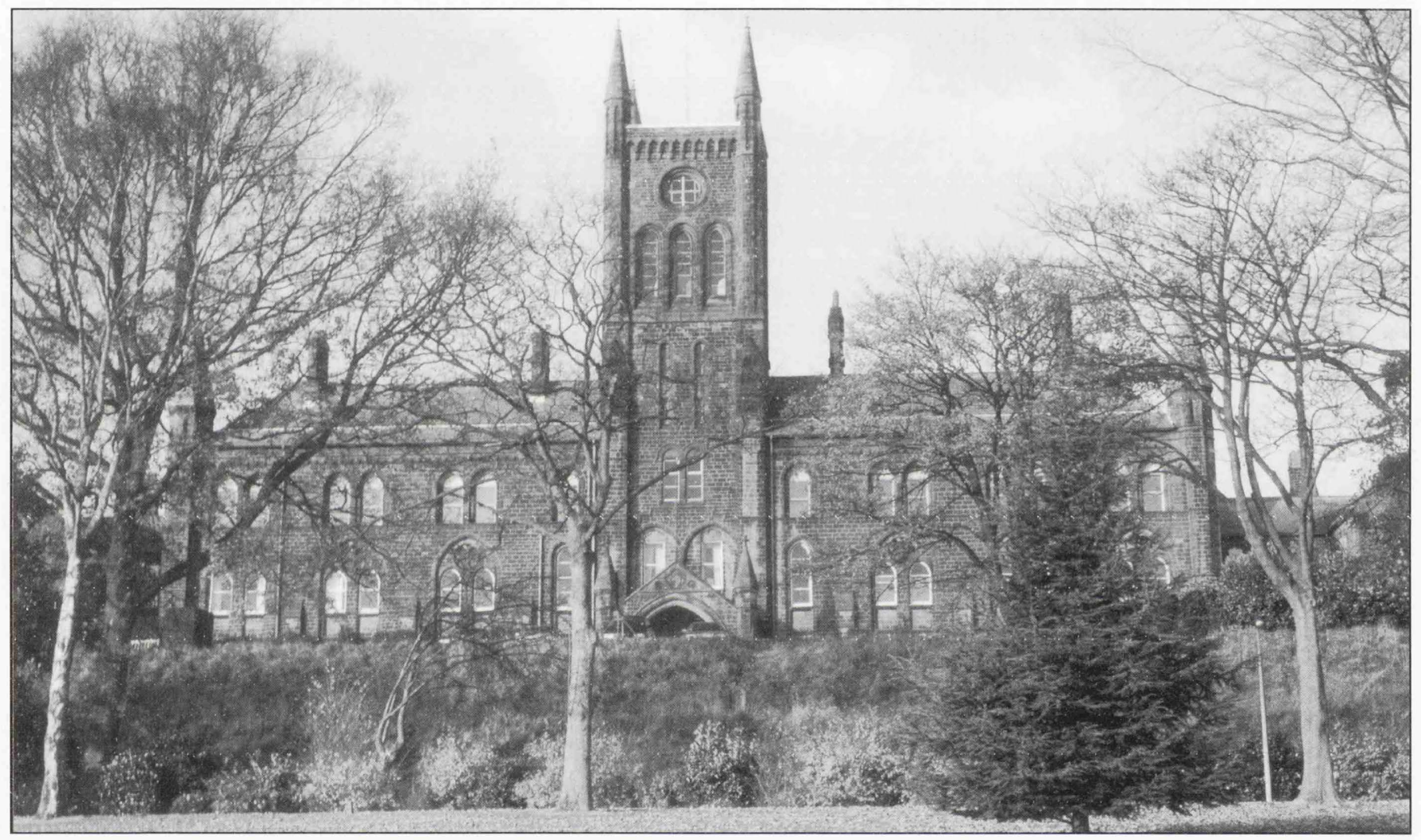

Lancaster Moor Hospital

Injuries involving fractures have occurred to four men and sixteen women; one woman dislocated a shoulder and another sustained a ruptured eyeball, both in falling on the way to the lavatory. Two of the other injured women were knocked down by fellow patients and one woman injured her arm by an impulsive movement in a lift. All remaining injuries were the result of accidental falls. Ten of the fractures occurred in patients over the age of 60 . It is a matter for much satisfaction that not one of these injuries proved fatal.

Since the last visit 174 patients have died (68 M, $106 \mathrm{~F})$; fifty were over the age of 70 . Post-mortem examinations were carried out on $41.3 \%$ of the deaths. In no case was there a bed sore.

The death rate for 1937 was 71 per thousand among men and 53 among women, the latter figure being rather lower than the average for other mental hospitals.

Good provision is made for the supply of special articles suitable for sick diet. There is a regular weekly issue of fresh fruit for all patients throughout the year. The improved character of the garden produce has ensured the issue of a
The nursing staff numbers 147 men and 313 women, seven of former and 28 of the latter being of charge rank; nine women nurses are employed on the male side.

We are glad to learn that steps have been taken to encourage members of the staff to qualify for the certificate. At present only $30 \%$ of the men and $13 \%$ of the women ward staff are certificated in mental nursing. We noticed that the nurses' lecture room is provided with chairs, not desks on which notes can be written.

There is a nucleus of a medical library for the use of the staff; a provision of some medical periodicals would be of considerable advantage to the work of the Hospital.

Dr Silverston has the assistance of Dr Wadsworth as Deputy and the remaining medical staff consists of two senior medical officers and four assistant medical officers

We have to thank Dr Silverston for every assistance during the course of our visit." 